\title{
The Size of the Finest Organic Structures*
}

\section{How Magnitudes Beyond the Resolving Power of Our Best Microscopes Are Estimated}

By Prof. Dr. W. Stempell

Minute anatomy has provided a fascinating subject for biological study ever since the introduction of the microscope. The resolving power of the microscope, however, cannot by any means whatever be increased beyond a certain limit set by the very nature of light. For this reason actual observations have at times been suplemented by more or less speculative reasoning based on the hypothetical size of the protein molecule. In this way it has been argued that organisms containing several thousand such protein molecules must measure at least 0.05 to $0.1 \mu \quad(1 \mu=1$ micromillimeter $=1 / 1,000$ millimeter $=$ about $1 / 25,000$ inch $)$. This would place them among magnitudes not far removed from those just discernible with the microscope, whose extreme limit of resolving power may be put at $0.2 \mu$, say. ${ }^{1}$

But all calculations of this kind labor under the disadvantage, that they are based on a hypothetical figure -the minimum size of a protein molecule-which is rather arbitrarily fixed, as we have at best only a very uncertain knowledge of it. It would be much more satisfactory if we could use for the basis of our calculation some actually measured structure.

As regards the resolving power of the microscope, if $e$ is the distance apart of two features which the microscope can just separate, and if $\lambda$ is the wave-length of the light employed, and if $a$ is the numerical aperture the light employed, and if $a$ is the numerical aperture
of the objective, then $e$ is connected with $\lambda$ and $a$ b of the objectiv
the relation.

$$
e=\frac{\lambda}{a}
$$

With ordinary light and a numerical aperture of 1.30 we have $e=0.42 \mu$. With oblique illumination, in whic case

\section{$e=\frac{\lambda}{2 a}$}

the value of $e$ is further reduced to $0.21 \mu$. With numerical aperture of 1.40 this can be brought down to $0.19 \mu$. Further reduction can only be secured by using ultra-violet light of short wave-lengths, in which case of course direct vision has to be abandoned and re placed by photographic observation. With oblique illuplaced by photographic observation. With oblique illu-
mination and cadmium light of wave-length $\lambda=275 \mu \mu$ mination and cadmium light of wave-length $\lambda=275 \mu \mu$
the distance between two points just separated can the distance between two points just separated can
finally be reduced to about $0.11 \mu$. This then represent practically the extreme limit of resolving power of the modern microscope.

An object particularly well adapted to serve as a standard of measurement is the spore of microsporidia, an excessively minute animal parasite. These spores

* Translated for the Scientific AMERICAN Supplement from Prometheus.

${ }^{1}$ Ct. Benecke, Bau und Leben der Bakterien, 1912, pp. 44 45. See also
1913 , p. 162

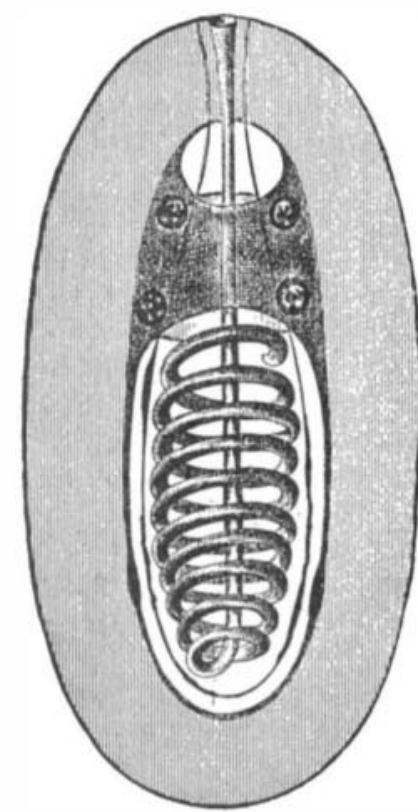

Fig. 1.-Diagram of the Internal Structure of a Spore of Nosema (Magnified 20,000 Times)

The darkly shaded portion at the top is a mass of protoplasm with four nuclei. Through the center of this passes the thread or flagelum, which is colled within the hollow spore, but can be projected outwat, are esrg-shaped and contain a coiled up thread which, under certain conditions, is projected outward, bein bodily turned inside out in the process. (See Fig. 1.) In the species known as N•sem bømbycis Nügeli, th germ of the silk worm disease, this thread, when protruded, has a length of $34 \mu$, and it has been definitely determined by microphotography in ultra-violet light, that the thread, when coiled up within the spore, has

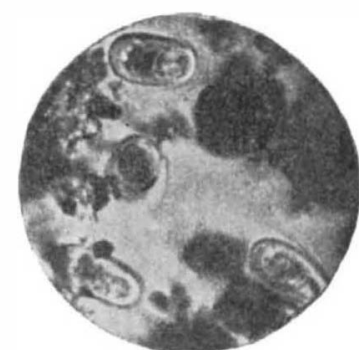

Fig. 2.-A Number of Spores of Nosema Photographed by Ultra-violet Light.

ten windings, its diameter being just about $1 \mu(1 / 25,000$ of an inch). This fact can be checked directly from the photograph reiresented in the accompanying illustration, Fig. 2. In that photograph the space occupied by the spiral thread measures 8 by 4 millimeters, and, as the object is magnified 3,600 times, this correspond to an actual dimension of 2 by $1 \mu$. It shows about 19 alternate black and white lines, corresponding to the 10 coils of the spiral and the spaces between them.
leach of these measures about 0.4 millimeter in the photograph, or $0.1 \mu$ in the object. The result of this measurement is further substantiated by the computation of the thickness of the thread from its length when projected to its full extent, and the space occupied by it in the interior of the spore. The entire spore is $4 \mu$ long and $2 \mu$ thick. The thickness of its envelope is $0.5 \mu$. Hence the space within the envelope measures $3 \mu$ at its greatest and $1 \mu$ at its smallest diameter. About one third of this space is occupied by a mass of protoplasm (see F'ig. 1, the darkly shaded portion), and there is thus left for the coiled up thread at most a cylindrical space $2 \mu$ long and $1 \mu$ in diameter. By measurement of an extended thread it is found to be $34 \mu$ long. From the space available in the interio of the spore it follows that the diameter of each coil can be at most $1 \mu$, hence its circumference at most is $3 \mu$, and therefore there can be at most ten windings in each coil.

Now this thread is actually a tube, which is turned inside out in the process of extrusion. Its wall, therefore, even if we allow nothing at all for the space within the tube, can at most have a thickness of $0.05 \mu$. In other species it can be shown that the wall of the thread can be no thicker than $0.008 \mu$

It will therefore be seen that among organisms there are structures of dimensions much smaller than have hitherto been suspected by bacteriologists. The demonstration of this is of considerable interest both to the biologist and to the physical chemist. The biological interest is derived from the fact that it is rendere extremely probable that organisms exist of such small size that we, with our most refined optical means, are not in a position to detect them. ${ }^{2}$

This accounts for the fact that it has been impossible hitherto to discover by the aid of a microscope the germ of certain infectious diseases.

To the physical chemist the matters here discussed are of interest owing to their bearing upon the question of molecular dimensions. For, as a matter of fact, the smallest of the dimensions mentioned above come very near to the dimensions of some of the larger molecules, such as for instance that of soluble starch. which has been estimated at $0.005 \mu$ by Lobry Debruyn. which has been estimated at $0.005 \mu$ by Lobry Debruyn.
It is not probable that the wall of the thread of the It is not probable that the wall of the thread of the
Nosema consists of a single layer of molecules; there Nosema consists of a single layer of molecules; there
are no doubt at least three such layers. This would give for the protein molecules a diameter of $0.0025 \mu$ Assuming a specific gravity of unity, this would give for the molecular weight of protein a value of 500,000 . This represents a maximum. In point of fact there are probably more than three layers of molecules, and

'Compare Prof. Behchold's article on "Ultra-filtration" in the $\mathbf{S c}$. moreover the structures here discussed are probably not the smallest which occur in nature. Therefore, the actual size of the protein molecule is probably considerably smaller than the figure indicated, i. e., 500,000.

\section{An Experiment for Showing Lines of Force in an Electrostatic Field}

Lveryone is familiar with the method of mapping a magnetic field of force by the aid of iron filings, which may, if desired, be made permanent by receiving the filings uron a suitable adhesive support, such as wax softened by heat.

More difficulty is experienced in mapping an electric field of force, but the following method, described by 3. M. Neville in Nature, provides a simple means for the purpose.

The method consists simply in allowing a scrap of cotton-wool to fall between the knobs of a Wimshurst machine, or among any conductors connected with them. As soon as the bit of fluff touches one of the conductors it moves off rapidly along a line of force. If the other conductor is oppositely charged the fluff will strike it, and again be repelled, usually in a slightly different direction, thus traversing a different line of force, and so on.

The scrap of charged fuff moves so rapidly under the electric forces that, owing to the persistence of vision, the shape of its path is very evident, and, owing to its lightness and the relatively great resistance offered by the air to its motion, its path approximates very closely indeed to the line of force.

\section{Electric Drill for Watch Makers}

THE mechanism shown in our illustration comprises a small electric motor and a pulley attached to the end of a bracket. The power is transmitted from the motor to the pulley and from the pulley to the drill spindle by means of a cord belt. By shifting a small lever the cord between the pulley and the drill holder can be slackened, whereby the latter is immediately brought to rest. The motor and pulley then continue to run empty and are ready at any instant to actuate the drill with the requisite speed as soon as the arresting lever is released.

The stem of the bracket which carries the pulley is constructed in telescopic fashion so that the tension of the cord can be adjusted at will. This is not only convenient in ordinary working, but also makes it unnecessary to determine with great exactness the length of the cord when renewing it, since the bracket can be adjusted to fit the cord.

The whole installation can be attached in a few - minutes to the working table, and thus forms a portable and very handy outfit. The motor is connected to



The Electric Watch Maker's Drill Attached to the Work Table.

the electric leads in the usual manner by means of flexible cord. The only accessory apparatus required is a contact breaker. The motor is constructed for all tensions up to 380 volts. 